\title{
Aspects on Patient Safety in Critical Care Unit
}

\author{
Hanan Mohammed Mohammed Assistant Professor of Medical-Surgical Nursing \\ Faculty of Nursing, Ain Shams University, Egypt
}

\begin{abstract}
:
Safety culture is a complex phenomenon that is not clearly understood by hospital leaders, thus making it difficult to operationalize. We found senior leadership accountability key to an organization-wide culture of safety. Ensuring patient safety is becoming increasingly important for intensive care unit practitioners. The intensive care unit is particularly prone to medical errors because of the complexity of the patients, interdependence of the practitioners, and dependence on team functioning. Workload, emotional stability, following safety precautions and lack of drug availability problems were found that lead to lack of the patients' safety in the ICU.
\end{abstract}

Keywords: Critical Care Unit, Patient safety 


\section{Tanta Scientific Nursing Journal}

\section{Introduction:}

Safety is a fundamental and essential attribute of quality health care. Patients Association revealed that only 45 per cent of National Health Service (NHS) organizations had patient safety as their first agenda item most of the time, with as average of 28 per cent of board meetings taken up by patient safety. National Patient Safety Agency (NPSA) nursing lead Woodward says it is essential to encourage a culture where health professionals can be open about patient safety and errors ${ }^{[1]}$.

According to the World Health Organization 2011 report on patient safety, health careassociated infections (HCAIs) are those that the patient gets infected with when they are admitted in the intensive care unit. HCAI is one of the main issue that hinders patient safety and this can lead to the patient been admitted for a longer time than should, been disabled for a long time, strain of finances, high expenses experienced by the patients and their families and also having too many deaths ${ }^{[2] \text {. }}$

Errors made with medications and patient safety are the major concern in the department of health. The surroundings in the ICU may be made worse by the presence of chronic diseases. The connection between safety and communication has been known for a long time. Nurses, doctors and other staff working in the ICU should be able to work together, and communicate in an effective way. This helps in the patient spending a shorter time in the ICU and less deaths occurring ${ }^{[3] \text {. }}$

Not communicating or lack of communication leads to problems with management and making of mistakes at the team level. Providing the workers with training makes their work of a high quality and due to this the potential errors are minimized. Communication with the other members of staff that are not professionals increases the potentiality of making errors. There is a strong relationship between providing patient safety in the ICU and the working relationship of the ICU care givers. Bad relationships lead to an increase in the errors and thus lead to bad outcome of the patients. Training of the teams using the resources available is a way to improve the relationships and thus enhancing patient safety ${ }^{[4]}$.

Nurses believe that caring aspects for example ethical concerns, patient relationships are essential to good nursing practice but day to day these factors play a secondary role. This is probably because of 


\section{Tanta Scientific Nursing Journal}

the dominance of technology and cure in the practice environment. During their first professional experience, nurses compare themselves with other nurses. Interpersonal relationships undergo rapid change and individuals take on new social roles. It is important for nursing care that professional nurses strive for patients to be cured of their illnesses and can detect care problems and potential complications as well as adjust care to individual patients ${ }^{[5] \text {. }}$

It is not easy to know for a fact if it is necessary to have the patient admitted in the ICU, if already is in the hospital. The individual who is responsible for deciding this is the surgeon or the person giving the anesthesia, has to balance the possibility of the patient losing their life from a situation that can be avoided on the usual ward versus spending a lot of resources once the patient is admitted in the ICU unnecessarily. How well the patient is monitored during the night is what decides whether their lives will be saved or not. The staff taking any action or not, when this notice that something is not right $^{[3]}$.

\section{Patient Safety in the ICU}

Patient safety is the prevention of errors and adverse effects to patients associated with health care. The care of critically ill patients is dependent on the use of complex medical equipment. Unfortunately, this equipment has the potential to develop faults, to be used incorrectly or to fail ${ }^{[6]}$.

Medication errors and patient safety are the most important in the health sector. The often environment of the ICU could be caused by the increase in chronic diseases, acuity of the patient and the advancement in technology and agents in the pharmaceutical agency. The guidelines in the professional sector are different on the way decisions should be made regarding the patient. The American Medical Association(AMA) advices the ethics committee or judicial review, whereas the American College of Physicians(ACP) recommends judicial review and the American Geriatrics Society(AGS) advises against the often involvement of the but advices that decisions be made by the doctors who are taking care of the patient ${ }^{[7] .}$

There is evidence that supports using of individual ways or processes to cut down on the incidences on errors in medications. Orders made through the computer by the people prescribing them has brought down the number of medication errors made in the case of failure in interpretation of the doctors' handwriting or the orders made 


\section{Tanta Scientific Nursing Journal}

verbally. Mistakes which involve administering of the wrong medication or giving medication to the wrong patient were brought down by $60 \%$ after the introduction of barcodes which match each and every patient's electronic orders and any other information that is related to medication. More to these strategies which are based electronically, using of pharmacies that use satellite and pharmacists that are unit-based help to improve safety by cutting down on the floor stock, which causes a possibility of causing medication errors ${ }^{[8]}$.

\subsection{Barriers to patient safety}

Nursing skill and staffing decisions are widely debated and contested. Managers argue that their mandate is to recruit and retain nurses and ensure that they are sufficiently trained to provide quality care within their competence level, thus enabling set work targets to be met. For their part, each nurse wants good working conditions, appropriate training, a fair workload, a safe environment and collegial support ${ }^{[9]}$.

No one would openly suggest that patient care should ever be compromised at the expense of a tight budget. Yet there is a risk of 'clinical compromise' that manifests itself on a daily basis as shift leaders struggle to make nurse-staffing decisions in their clinical setting. This tension is more in the ICU with its complex clinical, operational and governance requirements; its particularly vulnerable patient population; and its one-to-one nurse-patient relationship. There is limited evidence of the systems used in the ICU's for the processes used to assess individual nurse's skills and subsequent nurse-patient allocation decisions made (often thrice daily) at operational level ${ }^{[10] .}$

Contributing factors to inappropriate and potentially hazardous care delivery include the following. Nurses working out of their scope of practice in the ICU; nurses receiving inadequate orientation and workplace training; lack of adequate clinical and educational support systems in place; lack of knowledge of critical care nursing and therapies; nurses working in an unsafe ICU physical environment and nurses lacking awareness of occupational health and safety processes ${ }^{[6] \text {. }}$

Recognizing when a patient's condition is deteriorating is a key aspect of patient safety and the use of early warning scoring systems is integral to this. Compliance with such systems can often be poor. Observation taking is fundamental to patient care and is one of the most significant clinical skills 


\section{Tanta Scientific Nursing Journal}

undertaken by nurses. However, in recent years it appears to have become less of a priority for nurses and the skill is often delegated to junior nurses or support workers. It is documented nationally that recognition of deteriorating patients is poor and that the significance of ward-based observations is often overlooked ${ }^{[12]}$.

The National Patient Safety Agency (NPSA) analyzed 576 deaths that had been reported to the National Reporting and Learning System during 2005. The deaths could be interpreted as potentially avoidable and related to patient safety issues. It found that, of the 425 that occurred in acute/general hospitals, 64 were related to patient deterioration that had not been recognized or acted on ${ }^{[7] .}$

Early warning scores were devised to assist in the early detection of deteriorating patients. The scores are based on the premise that there is a common physiological pathway for deterioration in acutely unwell patients. Accurate and timely observations and adherence to early warning scoring systems are crucial in identifying those patients at risk of deterioration. Failure to do so could have devastating consequences not only for patients but also for trusts in terms of reputation ${ }^{[13] .}$
The Department of health has recommended the use of early warning systems as best practice for clinical observations. National Confidential Enquiry into Patient Outcome and Death (NCEPOD) further endorsed this recommendation, emphasizing that every inpatient should have early warning score recorded ${ }^{[9] .}$

National Institute for Health and Clinical Excellence (NIHCE) stated: 'Members of staff who are caring for the patients that are admitted in the critical hospital sectors should have a high standard in how they monitor, measure, interpret and act to the critically ill patient should be tested to make sure that they are able to show them. What should entail in this measurement scale is the heart rate, respiratory rate, temperature, how conscious they are, the amount of urine they produce, systolic blood pressure and the saturation of oxygen. A score is then gotten from the seven factors [11].

\subsection{Errors in the ICU related to patient safety}

The Institute of Medicine's 1999 report, To Err is Human: Building a Safe Health System, brought the problem of the errors in medicine out to the public. Debate in both public and professional sectors has showed that the exact morbidity and mortality due to 


\section{Tanta Scientific Nursing Journal}

medical errors is still in question. Regardless of the exact impact on patients, there is still recognition that errors in patients in the hospitals significantly increase morbidity and mortality. Although most of the hospitals have a number of policies and procedures that are in place to protect and care for patients, these policies may contain unappreciated "holes" that allow errors to slip through and also the results of interventions may not be delivered to caregivers in a timely fashion ${ }^{[14] .}$

The research done on the errors in medication and the race of having a safe administration of medication started in the 1960's in the United States of America. Errors in medication are defined as the mistakes that occur when medication is been prescribed, dispensed or administered be it that there is an error that occurred or not, is a major problem in health care sectors in the whole world. Most errors are possible to prevent but when they occur, apart from the high cost, there could be serious harm caused to the patient which could include them dying. In the ICU, it is more likely to make an error in medication because of the fact that there are many patients that are sick critically, who have medication prescribed and cause many times stress which is experienced by the staff who are at the same time been overworked at a place that is busy $^{[11]}$.

\section{Evidence-Based}

Evidence-based health care is the third subculture we identified. Healthcare organizations that demonstrate evidencebased best practices, including standardized processes, protocols, checklists, and guidelines, are considered to exhibit a culture of safety. Health care leaders refer to the aviation industry as a model for safety. Pilots use a standardized checklist before every flight to assure the aircraft, systems, and might crew are ready and working as designed. Interestingly, the World Health Organization recently introduced a standardized checklist recommended for use by the operative team before surgical procedures. Because the medical model of physician autonomy and the "art" of medicine is still prevalent, incorporating best practices and standardization may be leadership's greatest challenge to developing a culture of safety. However, as new generations of physicians are trained, the use of standardized guidelines may become more widely accepted $^{[15] .}$ 


\section{Tanta Scientific Nursing Journal}

\section{Patient-Centered}

Patient-centered culture is the seventh subculture we identified. A patient-centered culture embraces the patient and family as the sole reason for the hospital's existence. It promises to value the patient by providing a healing environment during the hospitalization and also to promote health and well-being as a continuum of care. The patient-centered hospital allows and empowers patients to be participatory in their care decisions. Leaders that share their patient-centered vision with their community allow the community to feel a sense of pride and ownership of their hospital. Patient stories can be used to put a "face" on system failures leading to potentially serious adverse events. Stories enhance the richness of description and create an atmosphere where discussion can lead to safety action ${ }^{[17,18,19] \text {. }}$

\section{Conclusion}

In the ICU, nurses, doctors and the subordinate staff care for the critically ill patients. They are mostly concerned about the quality of care that they give to their patients. It is good to work as a team and have a good communication as this helps in preventing the errors and also making better the outcome of the patients' and documenting as required. The ICU Safety Reporting System funded by the Agency for Healthcare Research and Quality is a strategy that gives way to volunteering, confidentiality and anonymously giving reports on the web of adverse events and errors that were about to happen. In 2006, the Agency for Healthcare Research and Quality and the Department of teamwork and communication are responsible to make sure that patients are given safe and quality care. It is been stressed that skills on how to communicate should be identified or the ways that can help in making sure that there is teamwork and communication, promoting safety and also improving the health of the patients.

\section{References}

[1] Adam S Standardization of nutritional support: are protocols useful? Intensive \& critical care nursing,2000; 283-289.

[2] Apold, J., Daniels, T., \& Sonneborn, M. Promoting collaboration and transparency in patient safety. Journal on Quality and Patient Safety, 2006;32(12): 672-675.

[3] Blake, S.C., Kohler, S., Rask, K., Davis, A., \& Naylor, D.V. Facilitators and barriers to 10 national quality forum 
safe practices. American Journal of Medical Quality2006; 21(5):323-334.

[4] J.H. Larrabee, C.L. Ostrow, M.L. Withrow, M.A. Janney, G.R. Hobbs, C. Burant Predictors of patient satisfaction with inpatient hospital nursing care Res Nurs Health2016; 27 (4): . 254-268

[5] D. Tschannen, B.J. Kalisch The impact of nurse/physician collaboration on patient length of stay $J$ Nurs Manag2012; 17 (7) : 796-803

[6]M. Riga, A. Vozikis, Y. Pollalis, K. Souliotis MERIS (Medical Error Reporting Information System) as an innovative patient safety intervention: a health policy perspective Health policy, 2015;119 (4): 539-548

[7] Clarke, J.R., Lerner, J.C., \& Marella, W. The role for leaders of health care organizations in patient safety. American Journal of Medical Quality2007; 22(5): 311-318.

[8] Cohen, M.M., Eustis, M.A., \& Gribbins, R.E. Changing the culture of patient safety: Leadership's role in health care quality improvement. Joint Commission Journal on Quality and Safety2003; 29(7): 329-335.

[9] Hansen, M.M., Durbin, J., SinkowitzCochran, R., Vaughn, A., Langowski,
M., \& Gleason, S. Do no harm:

Provider perceptions of patient safety. Journal of Nursing Administration2003; 33(10), 507-508.

[10]JKaissi, A. An organizational approach to understanding patient safety and medical errors. Health Care Manager2006; 25(4), 292-305.

[11] Frankel, A., Gandhi, T.K., \& Bates, D.W. Improving patient safety across a large integrated health care delivery system. International Journal for Quality in Health Care, 15(Suppl. 1), 31-40. [12] Yates, G.R., Bernd, D.L., Sayles, S.M., Stockmeier, C.A., Burke, G., \& Merti, G.E. (2005). Building and sustaining a systemwide culture of safety. Journal of Quality and Patient Safety, 2003;31(12), 684-689.

[12] Odwazny, R., Hasler, S., Abrams, R., \& McNutt, R. Organizational and cultural changes for providing safe patient care. Quality Management in Health Care2005; 14(3): 132-143.

[13] Nadzam, D., Atkins, P.M., Waggoner, D.M., \& Shonk, R. Cleveland clinic health system: A comprehensive framework for a health system patient safety initiative. 2005 
[14] Odwazny, R., Hasler, S., Abrams, R., \& McNutt, R. Organizational and cultural changes for providing safe patient care. Quality Management in Health Care2005; 14(3), 132-143.

[15]A.H. Rosenstein Original research: nurse-physician relationships: impact on nurse satisfaction and retention

Am J Nurs, 2002;102 (6): 26-34

[16]S. Thomson Nurse-physician collaboration: a comparison of the attitudes of nurses and physicians in the medical-surgical patient care setting Medsurg Nurs2017; 16 (2) :87-91104

[17]A.H. Rosenstein, M. O'Daniel Disruptive behavior and clinical outcomes: perceptions of nurses and physiciansAm J Nurs,2011; 105 (1): . 54-64

[18]L.A. Despins Factors influencing when intensive care unit nurses go to the bedside to investigate patient related alarms: a descriptive qualitative study Intensive Crit Care Nurs, 2017;4(3): 3

[20]C.S. Hartog, J. Benbenishty Understanding nurse-physician conflicts in the ICU Intens Ccare Med, 2015;41 (2): $331-333$
[19]J.M. Rothschild, C.P. Landrigan, J.W. Cronin, R. Kaushal, S.W. Lockley, E. Burdick, et al. The Critical Care Safety Study: the incidence and nature of adverse events and serious medical errors in intensive care Crit Care Med2009;, 33 (8): 1694-1700 